\title{
A CONSTRUCTION OF A SUBSPACE IN EUCLIDEAN SPACE WITH DESIGNATED VALUES OF DIMENSION AND METRIC DIMENSION
}

\author{
TATSUO GOTO
}

(Communicated by James West)

Dedicated to Professor Ryosuke Nakagawa on his 60th birthday

\begin{abstract}
For every integer $m, k$, and $n$ such that $0 \leq m \leq n-1$ and $m \leq k \leq \min \{2 m, n-1\}$, we construct a subspace $S_{m, k}^{n}$ in Euclidean $n$ space $\mathbf{R}^{n}$ satisfying the conditions that $\mu \operatorname{dim} S_{m, k}^{n}=m$ and $\operatorname{dim} S_{m, k}^{n}=k$, where $\mu$ dim denotes the metric dimension.
\end{abstract}

\section{INTRODUCTION}

Let $X$ be a subspace in Euclidean $n$-space $\mathbf{R}^{n}$ with $\mu \operatorname{dim} X=m, 0 \leq m \leq$ $n-1$, where $\mu \operatorname{dim}$ denotes the metric dimension. Then we have $m \leq \operatorname{dim} X \leq$ $\min \{2 m, n-1\}$ by Katětov's inequality $\operatorname{dim} X \leq 2 \mu \operatorname{dim} X$ [3].

In a previous paper [2] we constructed a subspace $S_{n, m}$ in $\mathbf{R}^{n}$ such that $\mu \operatorname{dim} S_{n, m}=m$ and $\operatorname{dim} S_{n, m}=\min \{2 m, n-1\}$. Thus the space $S_{n, m}$ is a subspace in $\mathbf{R}^{n}$ of metric dimension $m$ which has the maximal discrepancy with its covering dimension.

The purpose of this note is to prove the following theorem.

Theorem. Let $m, k$, and $n$ be arbitrary integers such that $0 \leq m \leq n-1$ and $m \leq k \leq \min \{2 m, n-1\}$. Then there exists a subspace $S_{m, k}^{n}$ in $\mathbf{R}^{n}$ such that $\mu \operatorname{dim} S_{m, k}^{n}=m$ and $\operatorname{dim} S_{m, k}^{n}=k$.

The space $S_{m, k}^{n}$ given below can be expressed as $S_{m, k}^{n}=S_{m}^{n} \cap N_{k}^{n}$, where $S_{m}^{n}$ denotes a space which is a slight modification of $S_{n, m}$ and $N_{k}^{n}$ denotes Nöbeling's $k$-dimensional space in $\mathbf{R}^{n}$.

\section{NotATION AND DEFINITIONS}

We denote by $\mathbf{Q}, \mathbf{Z}$, and $\mathbf{N}$ the set of rational numbers, integers, and positive integers, respectively. For a point $x=\left(x_{i}\right)$ in $\mathbf{R}^{n}$ we let $r(x)=$ $\operatorname{card}\left\{i: x_{i} \in \mathbf{Q}\right\}$. Then Nöbeling's $k$-dimensional space $N_{k}^{n}$ in $\mathbf{R}^{n}$ can be expressed as $N_{k}^{n}=\left\{x \in \mathbf{R}^{n}: r(x) \leq k\right\} \quad$ (cf. [1]).

Received by the editors December 3, 1991.

1991 Mathematics Subject Classification. Primary 55M10.

Key words and phrases. Dimension, metric dimension, Euclidean space, Nöbeling's space. 
For a metric space $X$ the metric dimension $\mu \operatorname{dim} X$ is defined as follows; $\mu \operatorname{dim} X \leq m$ iff for every $\varepsilon>0$ there exists an open cover $\mathscr{U}$ of $X$ such that mesh $\mathscr{U}<\varepsilon$ and ord $\mathscr{U} \leq n+1$ (cf. [1]).

The construction of the space $S_{n, m}$ in [2] is as follows. Let $T_{i}=\left\{t_{i, j}: j \in\right.$ $\mathbf{Z}\}, i \in \mathbf{N}$, be a set of real numbers such that:

$$
t_{i, j+1}-t_{i, j}=1 / i \text { for every } j
$$

and

$$
T_{i} \cap T_{i^{\prime}}=\varnothing \quad \text { if } i \neq i^{\prime} .
$$

For every $x \in \mathbf{R}^{n}$, we set $r_{i}(x)=\operatorname{card}\left\{j: x_{j} \in T_{i}\right\}, i \in \mathbf{N}$. Then the space $S_{n, m}$ in [2] can be written as

$$
S_{n, m}=\left\{x \in \mathbf{R}^{n}: r_{i}(x) \leq m \text { for every } i \in \mathbf{N}\right\} .
$$

In this construction we can assume that each $T_{i}$ is contained in $\mathbf{Q}$, and we denote by $S_{m}^{n}$ the space $S_{n, m}$ obtained by this modification.

\section{Proof of The Theorem}

We need a lemma due to Wilkinson [4].

Lemma. Suppose that $A_{1}, A_{2}, \ldots$ are closed proper subsets in $\mathbf{R}^{n}$ such that $\operatorname{dim}\left(A_{i} \cap A_{j}\right) \leq m$ whenever $i \neq j$. Then we have $\operatorname{dim}\left(\mathbf{R}^{n}-\bigcup_{i=1}^{\infty} A_{i}\right) \geq n-m-2$. Proof of the Theorem. By the definition

$$
S_{m}^{n}=\left\{x \in \mathbf{R}^{n}: r_{i}(x) \leq m \text { for every } i \in \mathbf{N}\right\} .
$$

Since $T_{i} \subset \mathbf{Q}$ for every $i$, it follows that $r_{i}(x) \leq r(x)$ for $x \in \mathbf{R}^{n}$, and hence $N_{m}^{n} \subset S_{m}^{n}$. Thus we have $N_{m}^{n}=S_{m, m}^{n} \subset S_{m, k}^{n} \subset S_{m}^{n}$, which implies that $m=\mu \operatorname{dim} N_{m}^{n} \leq \mu \operatorname{dim} S_{m, k}^{n} \leq \mu \operatorname{dim} S_{m}^{n}=m$ by [2, Lemma 4]. Thus we obtain

$$
\mu \operatorname{dim} S_{m, k}^{n}=m \text { for every } k, m \leq k \leq \min \{2 m, n-1\} .
$$

Moreover, we have $\operatorname{dim}\left(S_{m, k+1}^{n}-S_{m, k}^{n}\right) \leq 0$ because $S_{m, k+1}^{n}-S_{m, k}^{n}=S_{m}^{n} \cap$ $\left(N_{k+1}^{n}-N_{k}^{n}\right)$ and $\operatorname{dim}\left(N_{k+1}^{n}-N_{k}^{n}\right)=0$. Thus we obtain

(5) $\operatorname{dim} S_{m, k+1}^{n} \leq \operatorname{dim} S_{m, k}^{n}+1$ for every $k, m \leq k \leq \min \{2 m, n-1\}$.

We let $A_{i}=\left\{x \in \mathbf{R}^{n}: r_{i}(x) \geq m+1\right\}, i \in \mathbf{N}$. Condition (1) implies that each $A_{i}$ is the union of a countable locally finite family of $(n-m-1)$-dimensional planes. Hence, $A_{i}$ is closed in $\mathbf{R}^{n}$ and $\operatorname{dim} A_{i}=n-m-1$. From (3) it follows that $\mathbf{R}^{n}-S_{m}^{n}=\bigcup\left\{A_{i}: i \in \mathbf{N}\right\}$.

Case 1. $2 m \geq n-1$. In this case we have a sequence

$$
N_{m}^{n}=S_{m, m}^{n} \subset S_{m, m+1}^{n} \subset \cdots \subset S_{m, n-1}^{n} .
$$

In view of (5), to prove that $\operatorname{dim} S_{m, k}^{n}=k$ for every $k$ it suffices to show

$$
\operatorname{dim} S_{m, n-1}^{n} \geq n-1 \text {. }
$$

From (2) and the assumption that $2 m \geq n-1$, it follows that $A_{i} \cap A_{j}=\varnothing$ if $i \neq j$. Since $\mathbf{R}^{n}-N_{n-1}^{n}$ consists of a countable number of points and since

$$
\mathbf{R}^{n}-S_{m, n-1}^{n}=\left(\mathbf{R}^{n}-S_{m}^{n}\right) \cup\left(\mathbf{R}^{n}-N_{n-1}^{n}\right)=\bigcup\left\{A_{i}: i \in \mathbf{N}\right\} \cup\left(\mathbf{R}^{n}-N_{n-1}^{n}\right),
$$

we obtain (6) by the Lemma. 
Case 2. $2 m<n-1$. In this case we have a sequence

$$
N_{m}^{n}=S_{m, m}^{n} \subset S_{m, m+1}^{n} \subset \cdots \subset S_{m, 2 m}^{n} .
$$

It follows from (2) that $\operatorname{dim}\left(A_{i} \cap A_{j}\right) \leq n-2 m-2$ if $i \neq j$, because $2 m<n-1$. On the other hand, $\mathbf{R}^{n}-N_{2 m}^{n}$ is the countable union of $(n-2 m-1)$-dimensional planes $B_{j}, j \in \mathbf{N}$. Hence we have

$$
\mathbf{R}^{n}-S_{m, k}^{n}=\bigcup\left\{A_{i}: i \in \mathbf{N}\right\} \cup\left\{B_{j}: j \in \mathbf{N}\right\} .
$$

It is clear that, for every $i$ and $j, B_{j} \subset A_{i}$ or $\operatorname{dim}\left(A_{i} \cap B_{j}\right) \leq n-2 m-2$ and that $\operatorname{dim}\left(B_{j} \cap B_{j^{\prime}}\right) \leq n-2 m-2$ whenever $j \neq j^{\prime}$. Thus it follows from the Lemma that

$$
\operatorname{dim} S_{m, 2 m}^{n} \geq n-(n-2 m-2)-2=2 m .
$$

This implies that $S_{m, k}^{n}=k$ for every $k, m \leq k \leq 2 m$, by virtue of (5). This completes the proof.

\section{REFERENCES}

1. R. Engelking, Dimension theory, PWN, Warszawa, 1978.

2. T. Goto, Metric dimension of bounded subspaces of Euclidean spaces. I, Topology Proc. (to appear).

3. M. Katětov, On the relation between the metric and topological dimensions, Czechoslovak. Math. J. 8 (1958), 163-166. (Russian)

4. J. W. Wilkinson, $A$ lower bound for the dimension of certain $G_{\delta}$ sets in completely normal spaces, Proc. Amer. Math. Soc. 20 (1969), 175-178.

Department of Mathematics, faculty of Education, Saitama University, Urawa, JAPAN 